\title{
Ações de fotoeducação em saúde no ambiente escolar como estratégia para a prevenção de fotodermatoses
}

\author{
Luis Phillipe Nagem Lopes ${ }^{1}$, Agatha Cristiny Barros Bastos ${ }^{1}$, Luiz Eduardo Fileto Caldeira ${ }^{2}$, Fiammetta \\ Nigro $^{3}$, Zaida Maria Faria de Freitas ${ }^{4}$, Dalila dos Santos Cerqueira Pinto ${ }^{2}$, Márcia Maria Barros dos \\ Passos $^{1}$, Bárbara da Silva e Souza Lorca ${ }^{5}$, Elisabete Pereira dos Santos ${ }^{1}$
}

Resumo: Fotodermatoses são patologias cutâneas de origem multifatorial, que apresentam a exposição solar excessiva como o principal fator de risco, podendo causar neoplasias de pele. Ações educacionais, conhecidas como fotoeducação, são necessárias para conscientizar a população sobre a correta exposição ao sol e orientar condutas saudáveis em fotoproteção. O objetivo deste trabalho foi desenvolver um material educativo sobre fotoproteção e prevenção de fotodermatoses no ambiente escolar. Para tal, foi realizado um estudo qualitativo descritivo, baseado em relatos de experiências, sobre as atividades realizadas no ambiente escolar. Materiais informativos sobre as características gerais do câncer de pele, os medicamentos fotossensibilizantes, o uso correto do protetor solar e a adequada exposição solar foram elaborados após a interação dialógica entre os alunos de graduação de Farmácia e da Escola de Belas Artes, ambos da Universidade Federal do Rio de Janeiro, sob supervisão de farmacêuticos da Farmácia Universitária. Além disso, foi elaborado um quiz semiestruturado com perguntas e respostas e um quebra-cabeça, a fim de que os alunos fossem participantes ativos no processo de ensino-aprendizagem. A campanha foi realizada em escolas públicas, totalizando 230 alunos, que participaram de uma roda de conversa e dos jogos propostos. Ao final da campanha, notou-se que a ação educativa foi efetiva e com elevada participação dos alunos das escolas. Os materiais informativos elaborados com os jogos e rodas de conversas foram eficientes no esclarecimento das dúvidas e considerados ferramentas importantes no processo de aprendizagem dos jovens.

Palavras-chave: Câncer de pele, Fotoproteção, Promoção da saúde

Health photoeducation actions in the school environment as a strategy for the prevention of photodermatoses

Abstract: Photodermatoses are cutaneous pathologies of multifactorial origins, which have excessive sun exposure as the leading risk factor, causing skin neoplasms. Educational actions, known as photoeducation, are necessary to aware the population about the correct exposure to the sun and guide healthy behaviors regarding photoprotection. The work aimed at developing educational material about photoprotection and the prevention of photodermatoses in the school environment. Thus, a qualitative descriptive study was carried out, based on experiences about the school environment's activities. Informative materials about skin cancer's general characteristics, photosensitizing drugs, correct use of sunscreen, and adequate sun exposure were created after the dialogical interaction between undergraduate students of Pharmacy and Fine Arts School, both from the Federal University of Rio de Janeiro, under the supervision of pharmacists from the University Pharmacy. Also, a semi-structured quiz with questions and answers, and a puzzle was elaborated so that the students were active participants in the learning process. The campaign was carried out in public schools, with 230 students who participated in a conversation circle and the proposed games. At the end of the campaign, the educational action had a high participation rate and was considered effective. The informative materials developed, together with games and conversation circles, were efficient in clarifying doubts and considered important tools in the learning process of the young people.

Keywords: Skin cancer, Photoprotection, Health promotion
Originais recebidos em

02 de junho de 2020

Aceito para publicação em

14 de outubro de 2020

1

Universidade Federal do Rio de Janeiro (UFRJ), Faculdade de Farmácia.

2

Universidade Federal do Rio de Janeiro (UFRJ), Escola de Belas Artes.

3

Universidade Federal do Rio de Janeiro (UFRJ), Instituto de Macromóleculas.

4

Universidade Federal do Rio de Janeiro (UFRJ), Faculdade de Farmácia.

zaida@pharma.ufrj.br (autora para correspondência) 5 Fundação Centro Universitário Estadual da Zona Oeste (UEZO), Curso de Farmácia. 


\section{Introdução}

As fotodermatoses são definidas como patologias cutâneas originadas ou motivadas pela exposição à luz solar, determinando modificações de caráter inflamatório ou degenerativo, que podem repercutir em neoplasias de pele (Araújo et al., 2016). A origem dessas patologias é multifatorial, sendo a exposição demasiada e prolongada ao sol considerada o fator de risco primordial (Oliveira et al., 2020). A neoplasia cutânea, forma mais frequente e previsível de todos os cânceres, é dividida em dois grandes grupos, melanoma e não melanoma (Loo et al., 2020). Ambas podem ser evitadas pela proteção mecânica, com o uso de roupas, chapéus e óculos de sol, e pela proteção química, com a aplicação de protetor solar, já que o agente etiológico principal é a incorreta exposição ao sol (Araújo et al., 2016).

O câncer de pele não-melanoma é a neoplasia de maior ocorrência no Brasil, devido ao predomínio do clima tropical e alta incidência de radiação solar (Zink, 2014; Pires et al., 2018), correspondendo a cerca de 30\% de todos os tumores malignos registrados no país. Atualmente, são estimados mais de 85 mil casos novos entre os homens, e mais de 95 mil entre as mulheres. Esses valores correspondem a um risco estimado de 80,12 novos casos a cada 100 mil homens e 86,66 para cada 100 mil mulheres. O estado do Rio de Janeiro responde por $11,9 \%$ dos casos da doença no país, com $47,84 \%$ do total concentrados na capital (Instituto Nacional de Câncer José Alencar Gomes da Silva [INCA], 2020). Apesar de o câncer não-melanoma ser o tipo mais comum e ter baixa taxa de letalidade, as estatísticas de óbito quando o melanoma é diagnosticado tardiamente são elevadas, devido a seu alto potencial metastático (Zink, 2014; INCA, 2020).

Além da exposição excessiva à radiação solar, outros fatores podem aumentar o risco de desenvolver câncer de pele, como: pele, olhos e cabelos claros; histórico familiar ou pessoal de câncer de pele; sistema imune debilitado por doenças; e/ou o uso de medicamentos fotossensibilizantes, que aumentam a absorção da luz ultravioleta através da pele (azatioprina, ciclosporina, metoxisaleno, hidroquinona e sulfato de hidroxicloroquina) (American Cancer Society, 2017).

A fisiopatologia das neoplasias de pele é complexa e caracteriza-se pelo crescimento desordenado de células que invadem os tecidos e órgãos, podendo produzir metástase (Zink, 2014). Entretanto, apresenta uma forma de prevenção relativamente simples: a exposição correta ao sol (Araújo et al., 2016). Nesse cenário, ações de educação em saúde, baseadas na faixa etária do público, são fundamentais para a redução de casos de câncer de pele no Brasil.

O Ministério da Saúde define educação em saúde como "Processo educativo de construção de conhecimentos em saúde que visa à apropriação temática pela população (Falkenberg et al., 2014). Conjunto de práticas do setor que contribui para aumentar a autonomia das pessoas no seu cuidado e no debate com os profissionais e os gestores, a fim de alcançar uma atenção de saúde de acordo com suas necessidades". No cenário da prevenção de doenças de pele ocasionadas pela exposição incorreta ao sol, outro termo bastante empregado é a fotoeducação, que pode ser definida como "um conjunto de ações de caráter educativo, desenvolvido para conscientizar determinado grupo populacional sobre os riscos da exposição inadvertida ao sol e orientar condutas saudáveis em fotoproteção". Em ambas as definições, salientam-se três segmentos de atores prioritários: os profissionais de saúde, valorizando a prevenção e promoção tanto quanto as práticas curativas; os gestores, apoiando esses profissionais; e a população, construindo seus conhecimentos e aumentando sua autonomia nos cuidados, individual e coletivamente (Falkenberg, et al., 2014).

Focando na população que necessita construir seus conhecimentos e aumentar sua autonomia nos cuidados, individual e coletivamente, o público jovem tem um importante papel. Sobretudo, devido ao fato de que os jovens, em particular, constituem um grupo vulnerável à exposição solar inadequada, seja pela influência de 
fatores estéticos, seja pela maior frequência de atividades físicas ao ar livre (Pires et al., 2018; Patel et al., 2019). Logo, torna-se fundamental a estratégia de trabalho de profissionais da saúde para sensibilização deste público, despertando interesses e curiosidades sobre as práticas educativas, dinâmicas interativas e informações relacionadas à saúde (Galvão et al., 2003). Diante disso, a escola pode ser considerada um ambiente estratégico para a ampliação do conhecimento entre os jovens, já que é o local onde adquirem consciência sobre a sociedade e valores que norteiam suas escolhas, além de exercerem a sua máxima participação (Pires \& Branco, 2008). Por meio da elaboração de atividades estratégicas pautadas no aprendizado mútuo, busca-se a construção coletiva de conhecimentos, que contribua para a formação de professores e estudantes no contexto da saúde, promovendo o exercício da cidadania.

Dessa maneira, o projeto "Fotoeducação em saúde: câncer de pele, como evitar?" representa uma ferramenta para a promoção de saúde e prevenção de doenças, já que as atividades em saúde no ambiente escolar podem levar os jovens a ter maior percepção dos riscos associados à exposição solar sem proteção, bem como a terem ações mais reflexivas relacionadas à sua proteção e de seus familiares.

Nesse artigo, pretende-se abordar o processo de educação em saúde empregando o uso de tecnologias educativas, mediadas pela equipe de saúde da Farmácia Universitária da UFRJ (FU-UFRJ), junto a adolescentes em escolas do Ensino Médio (EM) na Zona Oeste do Rio de Janeiro.

\section{Metodologia}

\section{Desenho do estudo}

Trata-se de um estudo descritivo qualitativo, tipo relato de experiência (Araújo et al., 2013), baseado nas atividades realizadas com alunos do EM de escolas públicas do Rio de Janeiro, desenvolvidas no Projeto de Extensão "Fotoeducação em saúde: câncer de pele, como evitar?" da FU-UFRJ. Para a realização deste trabalho, foi montada uma equipe executora constituída por discentes e docentes do curso de Farmácia, uma aluna de pós-graduação do Instituto de Macromoléculas Professora Eloisa Mano, alunos da Escola de Belas Artes, além de farmacêuticos da Farmácia Universitária, todos pertencentes à Universidade Federal do Rio de Janeiro. Estes últimos foram responsáveis pelas ações nas escolas. Como projeto de extensão, suas diretrizes compreenderam: interação dialógica, interdisciplinaridade e interprofissionalidade; impacto de transformação social; indissociabilidade entre ensino, pesquisa e extensão; e impacto na formação do estudante. Todas foram observadas e postas em execução a cada etapa do desenvolvimento deste projeto. Para elaboração do material educativo, foi realizada uma revisão sobre o tema na literatura científica e, em seguida, elaborado um material informativo ilustrado.

\section{Levantamento bibliográfico e produção do material}

Inicialmente, os alunos de graduação do curso de Farmácia realizaram levantamento bibliográfico nas principais bases de dados, obtendo informações sobre medidas fotoprotetoras, como: fotoeducação, fotoproteção, fotodermatoses, exposição inadequada ao sol e uso correto de fotoprotetores. Essas informações foram supervisionadas pelo farmacêutico da farmácia universitária e repassadas para alunos extensionista da Escola de Belas Artes da UFRJ, a fim de elaborarem atividades lúdicas e criativas para o público jovem, objetivando gerar uma unidade visual com estética criativa, acessível e de fácil entendimento. De acordo com Melo (2005), aprender e ensinar brincando possibilita a socialização e troca de experiências, de conhecimento e de reflexão sobre as ações. As atividades lúdicas ativam o pensamento e a memória, além de gerar oportunidades de expansão das emoções, das sensações de prazer e da criatividade. Optou-se pela produção de materiais informativos autoexplicativos, contendo figuras e orientações sobre o uso do protetor 
solar, medicamentos fotossensibilizantes, e outros temas voltados à correta exposição solar. Além disso, foi elaborado um quiz semiestruturado, com perguntas e respostas, e um quebra-cabeça, ambos a serem aplicados aos alunos do EM, a fim de que fossem participantes ativos no processo de ensino-aprendizagem.

Todo o processo criativo dos alunos da Escola de Belas Artes foi acompanhado pelos alunos de Farmácia, proporcionando, desta forma, uma troca de saberes. Esta interação entre as áreas provocou mudanças nos alunos de ambos os cursos, o que foi possível observar posteriormente em outros trabalhos.

\section{Treinamento com os alunos de graduação}

Foram realizados treinamentos com os alunos de graduação, abordando a adequação da linguagem, as práticas de conscientização sobre os riscos da exposição ao sol, e o correto uso do protetor solar, visando estabelecer uma abordagem homogênea em saúde ao longo das atividades desenvolvidas nas escolas públicas estaduais da Zona Oeste da cidade do Rio de Janeiro.

Além disso, foram realizadas rodas de conversas entre os alunos de graduação, com a finalidade de esclarecer dúvidas, discutir mitos e verdades sobre o tema, bem como levantar ideias que tornassem a ação nas escolas de EM efetiva para o público jovem.

\section{Campanha realizada}

A ação de saúde nas escolas iniciou-se com a equipe apresentando o conteúdo proposto dentro do escopo de fotoeducação, fotodermatoses e prevenção de doenças da pele ocasionadas pela exposição incorreta ao sol. Em seguida, os alunos do EM participaram de uma roda de conversa com a equipe de saúde apontando dúvidas e curiosidades. Por fim, participaram dos jogos interativos propostos, quebra-cabeça e quiz, para a fixação do conteúdo (Figura 1A e 1B).

\section{Resultados e Discussão}

Um banner divulgador da Campanha "Fotoeducação: Câncer de pele, como evitar?" e informativos sobre medicamentos fotossensibilizantes e sobre o uso correto do protetor solar foram produzidos. Este último apresentou informações, como: a quantidade correta de produto que deve ser aplicada no corpo, o fator de proteção solar (FPS) adequado para os diferentes tipos de pele e o correto modo de aplicação do produto. Também foram produzidos jogos interativos, como um quebra-cabeça e um quiz (Figura 1C-F).

As ações de saúde, realizadas em formatos de campanhas educativas, foram desenvolvidas com 120 alunos do EM do Instituto de Educação Sarah Kubitschek, e 110 alunos do EM do Colégio Estadual Mário Quintana, totalizando 230 alunos.

A exposição à radiação ultravioleta (UV) proporciona variados efeitos benéficos à saúde, como a sensação de bem-estar físico e mental, o estímulo à produção de melanina e a produção de vitamina D3. Além disso, o tratamento de algumas doenças dermatológicas inclui a exposição regular à radiação UV, em conjunto com medicamentos adequados (Juzeniene \& Moan, 2012). No entanto, uma série de danos ao organismo pode ocorrer por ação da radiação UV, quando não são tomados os devidos cuidados à proteção da pele (Oliveira et al., 2020).

A radiação UV é a radiação mais energética da luz solar, possuindo grande poder de penetração na pele. Ela é capaz de promover reações químicas que envolvem transições eletrônicas, podendo gerar alterações genéticas nas células. A longo prazo, a radiação pode induzir alterações degenerativas nas células, tecidos fibrosos e vasos sanguíneos, e levar ao envelhecimento prematuro da pele (Watson et al., 2016; Krutmann et al., 2017). 


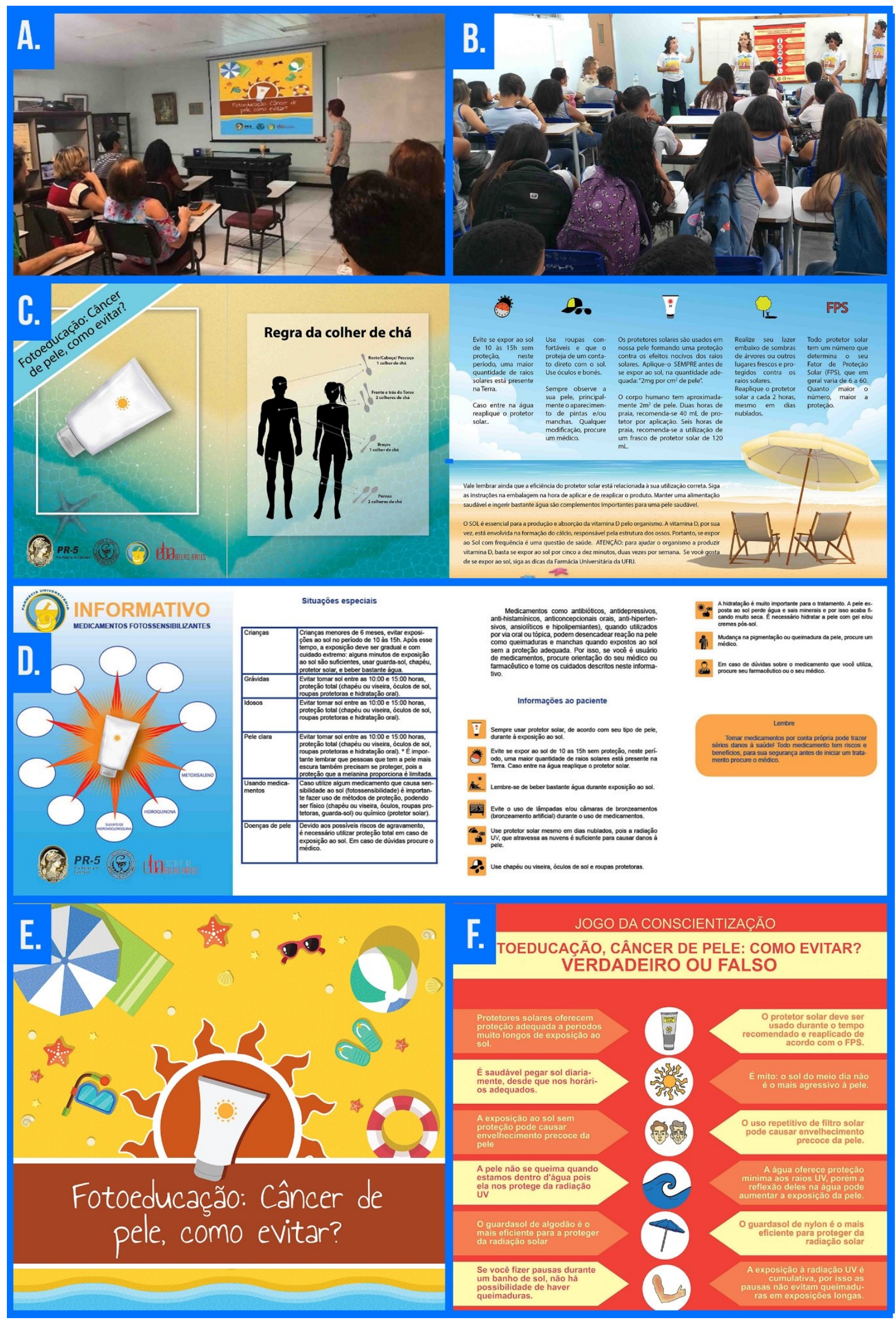

Figura 1. Atividades e Materiais desenvolvidos para a Campanha "Fotoeducação: Câncer de pele, como evitar?" A. Capacitação; B. Roda de conversa com os alunos do Ensino Médio; C- D. Informativos; E. Banner, F. Quiz. 
A radiação UV se divide em três faixas de energia distintas: UVA (320 a 400 nm), UVB (290 a 320 nm) e UVC (200 a 290 nm). A radiação UVA não é suficientemente energética para ser absorvida pelo ozônio, já a radiação UVB é absorvida pelo ozônio estratosférico, e a radiação UVC é completamente absorvida pelo ozônio e oxigênio da atmosfera (Kockler et al., 2012; Watson et al., 2016; Krutmann et al., 2017).

A radiação ultravioleta- $B$, que é atenuada pelo ozônio, é o fator mais importante na etiologia dos cânceres não-melanomas, denominados carcinomas (Carrara et al., 2019).

Vários fatores modificam a qualidade e quantidade da radiação recebida por uma superfície exposta em um ponto da terra: a estação do ano, a latitude, a altitude e a inclinação da incidência da energia solar. As variações da relação UVA/UVB são consequência disso, além da variação na quantidade absorbida pela espessura da atmosfera, para cada tipo de radiação (Watson et al., 2016).

A radiação UVB tem maior intensidade relativa e absoluta no verão, nos horários próximos ao meio-dia, nos locais próximos ao Equador e em altas altitudes. A alta velocidade do vento, a grande umidade relativa do ar e as altas temperaturas podem aumentar os efeitos do UVB (Corrêa, 2015; Watson et al., 2016). Superfícies como areia, neve e água são capazes de refletir a radiação da luz solar (Turner \& Parisi, 2018), provocando uma "dupla exposição" à pele.

A radiação UVB está associada com o bronzeamento e o câncer de pele, e atinge a superfície da Terra em pequenas quantidades. Por outro lado, a radiação UVA, além de potencializar o bronzeamento causado pelos raios UVB, tem maior incidência sobre a terra e penetra mais na pele (Krutmann et al., 2017). Também está presente o dia todo, enquanto a radiação UVB penetra menos e está presente nos horários entre 10 e 14 horas. Sendo assim, a radiação UVA é possivelmente responsável pelos maiores danos causados pela luz solar (Corrêa, 2015; Krutmann et al., 2017). Além disso, as radiações ultravioletas do tipo UVA, em especial as que apresentam comprimento de onda mais longos (340-400 nm), apresentam maior capacidade de penetrar na pele, atuando na formação de radicais livres no interior das células, e ocasionando o envelhecimento precoce após a contínua exposição (Krutmann et al., 2017).

Segundo o Instituto Nacional de Pesquisas Espaciais (INPE), a incidência dos raios ultravioletas é mais intensa entre 10 e 16h, período em que 70\% dos raios UV alcançam a superfície terrestre, inclusive na sombra, e sua radiação atinge o índice extremo ao meio-dia. Assim, os alunos do EM foram orientados que, antes de $10 \mathrm{~h}$ e após às 16h seriam os horários mais adequados para a exposição solar. A campanha demonstrou aos jovens que a exposição solar adequada é importante para a manutenção da saúde, além de ajudar a evitar o surgimento de fotodermatoses e neoplasias de pele.

Apesar dos esforços de campanhas educativas alertando sobre os efeitos maléficos da fotoexposição em horários de risco, isto é, entre 10 e 14h, observou-se que muitos alunos ainda optam pela exposição ao sol sem o protetor solar nos horários de riscos (Lages et al., 2012; Patel et al., 2019). Esse dado observado pela equipe de saúde do projeto se relaciona com um estudo transversal realizado com uma população de estudo jovem (Castilho et al., 2010). Esse estudo mostrou que 50,7\% de um total de 175 jovens, se expõem ao sol sem protetor solar no intervalo considerado de alto risco para efeitos lesivos do sol. Devido à faixa etária, é possível que esse comportamento esteja relacionado a atividades sociais, prática de esportes ao ar livre e intenção de aproveitar mais o dia (Pires et al., 2018; Patel et al., 2019).

O uso regular de fotoprotetor nas atividades gerais e durante a prática de esportes ao ar livre entre os estudantes ainda é limitado. Os alunos do EM demonstraram possuir um conhecimento limitado sobre o significado do Fator de Proteção Solar (FPS) nos produtos fotoprotetores, bem como sobre a regularidade adequada de seu uso. Quanto maior a percepção dos benefícios da fotoproteção, maior o FPS utilizado (Cokkinides et al., 2001). O uso correto do fotoprotetor, isto é, seu emprego diário, com aplicação 20 minutos 
antes da exposição solar e reaplicações a cada duas horas, é uma das medidas que devem ser constantemente estimuladas (Heerfordt et al., 2018).

Além do uso do produto fotoprotetor, a campanha também abordou a utilização de meios mecânicos de proteção contra a radiação solar, como: chapéu, barraca de sol, óculos e roupas com proteção UV. Os alunos do EM demonstraram conhecê-los, mas pouco utilizá-los. A proteção solar efetiva consiste na aplicação de fotoprotetores, além dos meios mecânicos de proteção e estratégias para evitar o sol, o que não é habitualmente praticado, mesmo quanto ao emprego da maneira mais corriqueira de proteção - uso do filtro solar (Araújo et al., 2016).

As informações sobre a associação entre exposição aos raios UV, câncer da pele e medicamentos fotossensibilizantes foram esclarecidas durante as atividades educativas nas escolas, mas, em concordância com outros trabalhos na literatura médica, nem sempre tal conhecimento reflete práticas adequadas de fotoproteção (Costa \& Weber, 2004; Szklo et al., 2007).

Atualmente, há inúmeros problemas que apresentam relação direta com a exposição solar. A falta de informação e sensibilização do público aos graves problemas de saúde tem atraído a atenção de um número crescente de pesquisadores em todo mundo. Consequentemente, o uso de fotoprotetor de amplo espectro passa a ser uma necessidade para garantir a saúde e a qualidade de vida da população. Analisando esse contexto, o acesso a esses produtos é um fenômeno que deve ser observado (Latha, 2013; Pires et al., 2018).

A ação de saúde desenvolvida não foi estruturada para analisar o perfil socioeconômico dos alunos, mas, segundo Batista et al. (2013), essa análise é de fundamental importância para elaboração de políticas públicas que garantam acesso a produtos visando a diminuição de casos de fotodermatoses e neoplasias. Sabe-se que o uso de produtos fotoprotetores está associado à renda familiar, visto que apresentam alto custo e não são fornecidos pelo Sistema Único de Saúde (SUS). Assim, o acesso ao produto e a adesão a práticas de prevenção são inferiores em famílias de baixa renda (Organização Pan-Americana da Saúde [OPAS/OMS], 2005).

Apesar da relação estabelecida entre exposição ao sol, fotodermatoses e câncer de pele, existem poucos estudos sobre os hábitos de proteção solar, especialmente no Brasil. Considerando que se trata de um país tropical, o risco de efeitos nocivos da exposição solar é elevado, principalmente devido ao efeito acumulativo, com um aumento no índice de câncer de pele no futuro (Batista et al., 2013). Ações de educação em saúde são fundamentais para que os hábitos de proteção sejam devidamente compartilhados com a população, com evidências científicas seguras e linguagem acessível ao público. Todas essas ações voltadas à educação e saúde da sociedade desenvolvidas na universidade são pontos importantes dentro das diretrizes estabelecidas para as atividades de extensão.

Cabe ressaltar que o processo formativo em saúde, desenvolvido com base em ações extensionistas, que se articula com o ensino e a pesquisa, induz a produção de novas práticas de cuidados conscientes e a formação integral, estando focado não apenas na aprendizagem técnica, mas também na ética, responsabilidade cidadã e compromisso social (Silva et al., 2013). Tal mecanismo desenvolve-se a partir das relações dialógicas entre sujeitos detentores de diferentes saberes, enriquecendo sobremaneira a experiência. Logo, a experiência extensionista não deve ser baseada na sobreposição do conhecimento científico sobre o conhecimento popular, mas ambos devem dialogar objetivando a atenção integral à saúde.

\section{Conclusão}

Com esse trabalho desenvolvido com adolescentes do ensino médio de escolas públicas, foi possível esclarecer dúvidas e demonstrar a necessidade de planos que minimizem o distanciamento entre a universidade e a comunidade. Espera-se que o projeto tenha gerado impactos positivos no estilo de vida desse público, 
conscientizando-o sobre a necessidade de utilização de produtos fotoprotetores e de meios mecânicos de proteção que, juntos, auxiliarão na prevenção de fotodermatoses e neoplasias. Também se espera que os alunos não só desenvolvam esses hábitos, mas também compartilhem com outras pessoas, criando uma rede de educação e autonomia do indivíduo no cuidado da sua saúde.

Quanto aos discentes da graduação, as ações desempenhadas contribuíram para suas formações, ampliando o comprometimento com a cidadania, a ética e a autonomia, e evidenciando que a troca de saberes dinamiza o conhecimento adquirido. Esse componente, inerente à dinâmica extensionista, possibilita à universidade cumprir com seu compromisso social e inserir o debate político no processo formativo em saúde.

Portanto, com a execução do projeto e sua campanha, foram obtidos resultados positivos. A ação educativa foi efetiva e com elevada participação dos alunos do EM durante todo o processo, além do evidente interesse em ações posteriores. $\mathrm{O}$ uso da linguagem adaptada ao público jovem foi fundamental para uma interação dialógica entre a universidade e os alunos das escolas públicas, valorizando a compreensão sobre como prevenir fotodermatoses e o uso correto de fotoprotetores, sendo a equipe da universidade convidada pelas direções das escolas a retornar com outras propostas. O empoderamento visando ao autocuidado foi fundamental para os jovens e também para a equipe do projeto, visto que foi um ambiente de troca e aprendizado, que muito contribuiu para a formação ética e cidadã dos alunos da graduação e do Ensino Médio.

\section{Contribuição de cada autor}

A.C.B.B., L.P.N.L., F.N., M.M.B.P., Z.M.F.F., E.P.S. participaram com contribuições substanciais na concepção e planejamento do projeto, na execução do projeto, na redação e revisão intelectual crítica do artigo; L.E.F.C., D.S.C.P. contribuíram na produção e desenvolvimento de ilustração e imagem, sendo todos responsáveis pela aprovação final do artigo para publicação, diante disso, solicitamos que a primeira autoria seja compartilhada entre esses autores. B.S.S.L. participou na articulação entre a universidade e escolas participantes e na revisão do manuscrito.

\section{Agradecimentos}

Os autores agradecem ao Programa Institucional de Fomento Único de Ações de Extensão (PROFAEX) da Universidade Federal do Rio de Janeiro - UFRJ pela concessão das bolsas de extensão, à direção da Faculdade de Farmácia pelo apoio e transporte para as escolas públicas.

\section{Referências}

American Cancer Society. About basal and squamous cell skin cancer. Atlanta: American Cancer Society. Recuperado de https://www.cancer.org/content/dam/CRC/PDF/Public/8818.00.pdf

Araújo, F. C., Sousa, B. R. M., Leite, G. G., Freitas, L. C., Lemos, E. L. C., \& Pires, C. A. A. (2016). Avaliação dermatológica de agentes comunitários de saúde sujeitos à fotoexposição em região tropical do Brasil. Scientia Medica, 26(4), 1-7.

Araújo, M. G., Cassiano, A. N., Holanda, C. S. M., Moreira, P. V. S. Q., \& Giovannini P. E. (2013). Educação em saúde no ensino infantil: metodologias ativas na abordagem da ação extensionista. Revista de Enfermagem UFPE on line, 7(1), 306-313.

Batista, T., Fissmer, M. C., Porton, K. R. B., \& Schuelter-Trevisol, F. (2013). Avaliação dos cuidados de proteção solar e prevenção do câncer de pele em pré-escolares. Revista Paulista de Pediatria, 31(1), 17-23. 
Carrara, I. M., Melo, G. P., Bernardes, S. S., Neto, F. S., Ramalho, L. N. Z., Marinello, P. C., ...., \& Cecchini, A. L. (2019). Looking beyond the skin: Cutaneous and systemic oxidative stress in UVB-induced squamous cell carcinoma in hairless mice. Journal of Photochemistry and Photobiology, 195, 17-26.

Castilho, I. G., Sousa, M. A. \& Leite, R. M. S. (2010). Fotoexposição e fatores de risco para câncer da pele: Uma avaliação de hábitos e conhecimentos entre estudantes universitários. Anais Brasileiros de Dermatologia, 85(2), 173-178.

Cokkinides, V. E. (2001). Sun exposure and sun-protection behaviors and attitudes among US youth, 11 to 18 years of age. Preventive Medicine, 33(3), 141-151.

Corrêa, M. P. (2015). Solar ultraviolet radiation: Properties, characteristics and amounts observed in Brazil and South America. Anais Brasileiros de Dermatologia, 90(3), 297-313.

Costa, F. B. \& Weber, M. B. (2004). Avaliação dos hábitos de exposição ao sol e de fotoproteção dos universitários da Região Metropolitana de Porto Alegre, RS. Anais Brasileiros de Dermatologia, 79(2), 149-155.

Falkenberg, M. B., Mendes, T, P. L., Moraes, E. P., \& Souza, E. M. (2014). Educação em saúde e educação na saúde: Conceitos e implicações para a saúde coletiva. Ciência \& Saúde Coletiva, 19, 847-852.

Galvão, M. T. G. (2003). Sexualidade e conhecimento das doenças sexualmente transmissíveis e AIDS entre adultos em um município do interior do nordeste brasileiro. Jornal Brasileiro de Doenças Sexualmente Transmissíveis (DST), 15(3), 37-40.

Heerfordt, I. M., Torsnes, L. R., Philipsen, P. A. \& Wulf, H. C. (2018). Sunscreen use optimized by two consecutive applications. PLoS One, 13(3), e0193916.

Instituto Nacional de Câncer José Alencar Gomes da Silva (2020). Rio de Janeiro. Estatísticas de câncer. Rio de Janeiro: INCA. Recuperado de https://www.inca.gov.br/numeros-de-cancer

Juzeniene, A., \& Moan, J. (2012). Beneficial effects of UV radiation other than via vitamin D production. Dermatoendocrinology, 4(2), 109-117.

Kockler, J., Oelgemöller, M., Robertson, S., \& Glass, B. D. (2012). Photostability of sunscreens. Journal of Photochemistry and Photobiology C: Photochemistry Reviews, 13, 91-110.

Krutmann, J., Bouloc, A., Sore, G., Bernard, B. A., \& Passeron, T. (2017). The skin aging exposome. Journal of Dermatological Science, 85(3), 152-161.

Lages, R. B. (2012). Detecção precoce do câncer de pele: Experiência de campanha de prevenção no Piauí-Brasil. Revista Brasileira em Promoção da Saúde, 25(2), 221-227.

Latha, M. S. (2013). Sunscreening agents: A review. The Journal of Clinical and Aesthetic Dermatology, 6(1), 16-26.

Loo, K., Soliman, I., Renzetti, M., Li, T., Wu, H., Reddy, S.,..., \& Farma, J. M. (2020). Impact of sun exposure and tanning patterns on next-generation sequencing mutations in melanoma. Journal of Surgical Research, 254, 147-153.

Melo, C. M. R. (2005). As atividades lúdicas são fundamentais para subsidiar o processo de construção do conhecimento. Information Filosófica, 2(1), 128-137.

Oliveira, P. T. N., Morégula, A. A., \& Silva, M. F. (2020). Correlação entre concepção de saúde e comportamento preventivo relativo à exposição solar. Brazilian Journal of Development, 6(3), 10404-10417.

Organização Pan-Americana da Saúde (2005). Avaliação da Assistência Farmacêutica no Brasil. Brasília: OPAS/OMS.

Patel, A. R., Zaslow, T. L., Wren, T. A. L., Daoud, A. K., Campbell, K., Nagle, K., \& Coel, R. A. (2019). A characterization of sun protection attitudes and behaviors among children and adolescents in the United States. Preventive Medicine Reports, 16, 100988. 
Pires, C. A. A., Fayal, A. P., Cavalcante, R. H., Fayal, S. P., Lopes, N. S., Fayal, F. P., \& Santos, M. A. L. (2018). Câncer de pele: Caracterização do perfil e avaliação da proteção solar dos pacientes atendidos em serviço universitário. Journal of Health \& Biological Sciences, 6(1), 54-59.

Pires, S. F. S., \& Branco, A. U. (2008). Cultura, self e autonomia: Bases para o protagonismo infantil. Psicologia: Teoria e Pesquisa, 24(4), 415-421.

Silva, A. F. L., Ribeiro, C. D. M., \& Silva Júnior, A. G. (2013). Pensando extensão universitária como campo de formação em saúde: Uma experiência na Universidade Federal Fluminense, Brasil. Interface-Comunicação, Saúde, Educação, 17, 371-384.

Szklo, A. S. (2007). Comportamento relativo à exposição e proteção solar na população de 15 anos ou mais de 15 capitais brasileiras e Distrito Federal, 2002-2003. Cadernos de Saúde Pública, 23(4), 823-834.

Turner J., \& Parisi A. V. (2018). Ultraviolet radiation albedo and reflectance in review: the influence to ultraviolet exposure in occupational settings. International Journal of Environmental Research and Public Health, $15(7), 1507$.

Watson, M., Holman, D. M., \& Maguire-Eisen, M. (2016). Ultraviolet radiation exposure and its impact on skin cancer risk. Seminars in Oncology Nursing, 32(3), 241-254.

Zink, B. S. (2014). Câncer de pele: A importância do seu diagnóstico, tratamento e prevenção. Revista Hospital Universitário Pedro Ernesto, 13(Supl. 1), 76-83.

\section{Como citar este artigo:}

Lopes, L. P. N., Bastos, A. C. B., Caldeira, L. E. F., Nigro, F., de Freitas, Z. M. F., Pinto, D. S. C., dos Passos, M. M. B., Lorca, B. S. S., \& dos Santos, E. P. (2020). Ações de fotoeducação em saúde no ambiente escolar como estratégia para a prevenção de fotodermatoses. Revista Brasileira de Extensão Universitária, 11(3), 397-406. https://periodicos.uffs.edu.br/index.php/ RBEU/article/view/11548/pdf 